\title{
Two pictures are better than one
}

Different imaging modalities always have unique strengths and weaknesses. The combination of two popular in vivo imaging methods shows that combining them gives you the best of both.

Researchers using conventional in vivo imaging techniques to examine biological processes in an intact living animal have generally had to choose between highly sensitive detection of probes with lackluster resolution and anatomical information, and highresolution anatomy but low probe detection sensitivity. These methods are epitomized by positron emission tomography (PET), which can detect picomolar concentrations of radiolabels, and magnetic resonance imaging (MRI), which provides detailed anatomical images but can only detect high probe concentrations. Simultaneous PET and MRI imaging would be a boon to researchers.

There are two choices for combining PET and MRI. The first is serial imaging in one device and then the other, but this makes it difficult to co-register images and impossible to perform temporal correlated studies. The second option is to combine the devices into one. Simon Cherry at the University of California at Davis has been working on this since the 1990s.

Combining these modalities is not easy. The detectors in conventional PET scanners are extremely sensitive to magnetic fields and degrade the MRI performance if they are in the MRI field of view. In 1997 Cherry's lab reported a brute-force approach to get around this problem and acquired the first simultaneous PET-MRI images. "This used massively long optical fibers to bring the signal out of the PET scanner to the electronics sitting far away from the magnets. It was very impractical but it did show the potential," says Cherry.

Around the same time Bernd Pichler was a graduate student investigating new photon detectors called avalanche photodiodes (APDs). APDs had a worse performance than the current photomultiplier-based PET detectors, but Pichler recognized that these

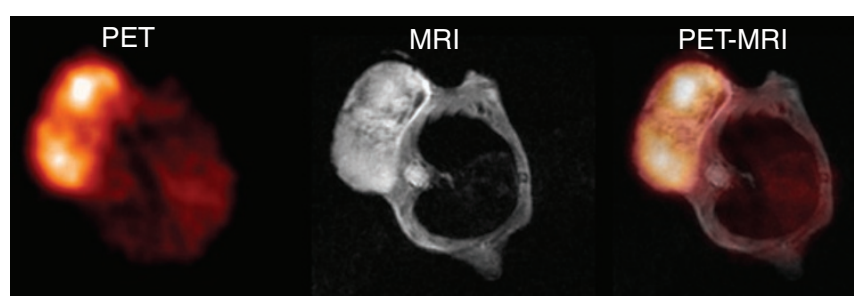

Figure 1 PET, MRI and overlay images of a $0.8 \mathrm{~cm}^{3}$ tumor in a mouse acquired simultaneously with PET-MRI using $\left[{ }^{18} \mathrm{~F}\right]$ FLT. Images courtesy of B. Pichler.

devices would not be very sensitive to magnetic fields and started sticking them into magnets to see what would happen. Pichler says, "I put my first APDs in a 9.4 Tesla magnet in 1997 and wrote a paper, which was rejected with the response that no one would be interested in PET-MRI."

Cherry was interested in Pichler's work though, and Pichler joined Cherry's group as a research scientist to help build a practical PET-MRI machine. Cherry says, "We came up with two related but different schemes. One retains a small amount of optical fiber coupling to minimize interference and a position-sensitive APD." The short optical fibers allow the detectors to be positioned outside the MRI field of view but still inside the machine. "The second approach was to ditch the fiberoptics completely and go with an array of single channel APDs," says Cherry. "I was pushing the limited fiberoptic approach, and Pichler was pushing the other approach. So we said, let's do both." Because of the tradeoff between signal loss from optical fibers and MRI interference from APDs located within the MRI field of view, Cherry recalls, "the gut feeling was our approach would be worse for PET but better for MRI, and his approach would be better for PET but worse for MRI."

They were unable to determine the full answer to this question before Pichler left the lab to take a position in Germany. Ciprian Catana in Cherry's lab took the lead in developing their approach while Pichler developed his at the University of Tübingen, and the results were published independently
(Catana, C. et al., 2008; Judenhofer, M.S. et al., 2008). Cherry says, "I think the important thing from the communities' point of view is that two independent systems have been built, and both are working very well. The MRI performance is effectively unperturbed with a few percent drop in signal-to-noise ratio in both approaches. The performance of the PET inserts is already comparable to what you had with a standalone preclinical PET scanner 7-8 years ago and in terms of spatial resolution matches that of today's state-of-the-art commercial scanners."

The ability to image whole living animals with PET and MRI simultaneously (Fig. 1) opens up a whole arena of applications, but Cherry believes the main one will be looking at two things and correlating changes over time. For example, drug metabolism, receptor expression or cell tracking could be monitored over time with high sensitivity, and precise information on localization and local tissue structure. MRI also allows easy correction of motion artifacts from respiration or cardiac activity.

Both papers show compelling preliminary data for several different applications. Both groups are hard at work using these systems for real biological studies.

\section{Daniel Evanko}

\section{RESEARCH PAPERS}

Catana, C. et al. Simultaneous in vivo positron emission tomography and magnetic resonance imaging. Proc. Natl. Acad. Sci. USA 105, 3705-3710 (2008).

Judenhofer, M.S. et al. Simultaneous PET-MRI: a new approach for functional and morphological imaging. Nat. Med. 14, 459-465 (2008). 